\title{
Modified Fincke-Pohst Algorithm for Low-Complexity Iterative Decoding over Multiple Antenna Channels
}

\author{
Haris Vikalo \\ Information Systems Lab \\ Stanford University \\ Stanford, CA 94305, USA \\ e-mail: hvikaloostanford.edu
}

\author{
Babak Hassibi \\ Department of Electrical Engineering \\ California Institute of Technology \\ Pasadena, CA 91125, USA \\ e-mail: hassibi@caltech.edu
}

Abstract - In recent years, soft iterative decoding techniques have been shown to greatly improve the bit error rate performance of various communication systems. For multiple antenna systems employing spacetime codes, however, it is not clear what is the best way to obtain the soft-information required of the iterative scheme with low complexity. In this paper, we propose a modification of the Fincke-Pohst (sphere decoding) algorithm [1] to estimate the maximum a posteriori (MAP) probability of the received symbol sequence. The new algorithm (FP-MAP) solves a nonlinear integer least-squares problem and, over a wide range of rates and SNRs, has polynomial-time (often cubic) expected complexity. The FP-MAP algorithm provides soft detection information for the soft channel decoder. The soft decoder's output is then fed back to the FP-MAP, and iterated on. The performance of the FP-MAP algorithm on a multiple antenna system employing turbo code is demonstrated.

\section{INTRODUCTION}

We assume a discrete-time block fading multiple antenna channel model with $M$ transmit and $N$ receive antennas, where the transmitted signal $\mathbf{s}$ and received signal $\mathbf{x}$ are related by

$$
\mathbf{x}=\mathbf{H} \mathbf{s}+\mathbf{v}
$$

where $\mathbf{H}$ is the channel matrix, and $\mathbf{v}$ is the additive noise vector. Furthermore, transmitted symbols $s$ are obtained upon modulating a coded sequence $\left\{c_{i}\right\}$ onto an m-dimensional $(m=2 M)$ subset of a lattice $\mathcal{D}_{L}^{m}$ (lattice subset $\mathcal{D}_{L}^{m}$ spans over $L$ points in each of $m$ dimensions).

For a known channel in AWGN, MAP detection is equivalent to the optimization problem

$$
\min _{s \in \mathcal{D}_{L}^{m}}\left[\|x-H s\|^{2}-\sum_{k=1}^{m} \log p\left(s_{k}\right)\right],
$$

where $p\left(s_{k}\right)$ denotes a priori probability of a $k^{t h}$ entry in a symbol vector. Applying the idea of Fincke-Pohst algorithm (which has polynomial expected complexity [2]), rather than to search over the entire lattice, we search only over lattice points $\mathbf{s}$ that belong to the geometric body described by

$$
r^{2} \geq(\mathrm{s}-\hat{\mathbf{s}})^{*} R^{*} R(\mathbf{s}-\hat{\mathbf{s}})-\sum_{k=1}^{m} \log p\left(s_{k}\right),
$$

where $R$ is lower triangular matrix following QR factorization of $H$ and $\hat{\mathrm{s}}$ is standard least-squares estimate. [Note that, unlike in the original sphere decoder algorithm, this geometric body is no longer a hypersphere.] The search radius $r$ in (2) can be chosen according to the statistical properties of the noise and a priori distribution of $\mathbf{s}$.

For an iterative decoding scheme, we require soft information, i.e., probability that each bit is decoded correctly. To this end, consider log-likelihood ratio of the form

$$
L\left(c_{i} \mid \mathbf{x}\right)=\log \frac{\sum_{s: c_{i}=+1} e^{-\|x-H s\|^{2}+\sum_{j} \log p\left[s_{j}\right]}}{\sum_{s: c_{i}=-1} e^{-\|\mathbf{x}-H \mathbf{s}\|^{2}+\sum_{j} \log p\left[s_{j}\right]}}
$$

Computing (3) over entire signal space $\mathcal{D}_{L}^{m}$ is of prohibitive complexity. Instead, we constrain ourselves to those $s \in \mathcal{D}_{L}^{m}$ for which argument in (1) is small. [Note that these are the signal vectors whose contribution to the numerator and denominator in (3) is significant.]

Assume that the search in (2) yields the set of points $\mathcal{S}=$ $\left\{s^{(1)}, s^{(2)}, \ldots, s^{(l)}\right\}$. The vector $s \in \mathcal{S}$ that minimizes $(1)$ is the solution to the MAP detection problem. The soft information for each bit $c_{i}$ can be estimated from (3), by only summing the terms in the numerator and denominator such that $s \in \mathcal{S}$.

Figure 1 shows the bit-error performance of the system with 4 transmit and 4 receive antennas, $16-Q A M$ constellation and parallel concatenated turbo code with rate $R=1 / 2$ and length 9216 information bits. For each iteration of the FPMAP, turbo (inner) decoder performs 8 iterations of its own.

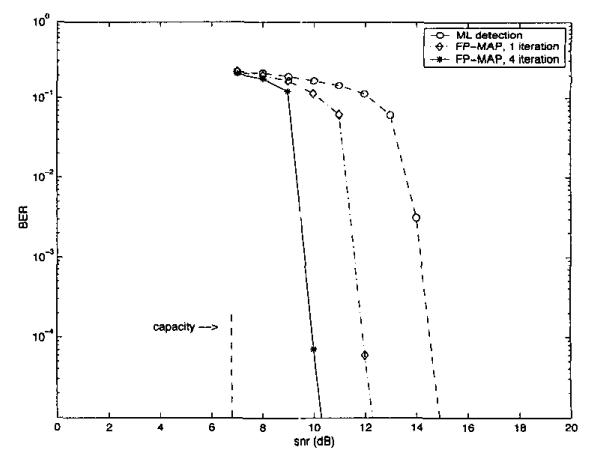

Figure 1: Rate 1/2 turbo code, $4 \times 4$ system, 16-QAM

ACKNOWLEDGMENTS

Thanks to Hui Jin for useful discussions.

\section{REFERENCES}

[1] U. Fincke and M. Pohst, "Improved methods for calculating vectors of short length in a lattice, including a complexity analysis," $M$ athematics of Computation, vol. 44, pp. 463-471, 1985.

[2] B. Hassibi and H. Vikalo, "Expected complexity of the sphere decoder algorithm," submitted to the IEEE Transactions on Signal Processing, 2002. 